Supporting information for:

\title{
The Reactivity of All-Metal Aromatic Complexes: a Theoretical Investigation on the Methane Activation Reaction
}

\author{
Xingbang $\mathrm{Hu}$, Haoran $\mathrm{Li}^{*}$ and Congmin Wang
}

Department of Chemistry, Zhejiang University, Hangzhou 310027, P. R. China

\section{List of Contents}

1) Table S1. Enthalpies changes (in $\mathrm{kcal} / \mathrm{mol}$ ) and free energies change (in $\mathrm{kcal} / \mathrm{mol}$ ) of the reaction pathways of methane oxidation listed in Figure 2.

2) Table S2. All vibrations of the transition states

3) The Z-MATRIX of the optimized structures

* Corresponding author. Fax: +86-571-8795-1895

Email Address: lihr@zju.edu.cn (H. Li) 
Table S1. Enthalpies changes (in $\mathrm{kcal} / \mathrm{mol}$ ) and free energies change (in $\mathrm{kcal} / \mathrm{mol}$ ) of the reaction pathways of methane oxidation listed in Figure 2.

\begin{tabular}{ccccc}
\hline & \multicolumn{2}{c}{$\mathrm{TS}$} & \multicolumn{2}{c}{ Product } \\
\hline & $\Delta^{\neq} \mathrm{H}$ & $\Delta^{\neq} \mathrm{G}$ & $\Delta \mathrm{H}$ & \\
\hline $\mathrm{O}_{2}-\mathrm{C}-\mathrm{T}$ & 111.9 & 119.8 & & \\
$\mathrm{Al}_{4} \mathrm{CaO}-\mathrm{I}-\mathrm{C}-\mathrm{TS} 1$ & 71.1 & 76.8 & & 63.6 \\
$\mathrm{Al}_{4} \mathrm{CaO}-\mathrm{C}-\mathrm{TS} 1$ & 67.6 & 74.4 & 59.8 & -56.2 \\
$\mathrm{Al}_{4} \mathrm{CaO}_{2}-\mathrm{C}-\mathrm{TS} 1$ & 52.5 & 57.8 & -54.9 & \\
$\mathrm{Al}_{4}{ }^{2-} \mathrm{O}_{2}-\mathrm{C}-\mathrm{TS} 1$ & 42.8 & 46.4 & & 11.7 \\
$\mathrm{Al}_{4} \mathrm{CaO}_{2}$-C-TS2 & 32.8 & 40.2 & 7.3 & -0.6 \\
$\mathrm{Al}_{4} \mathrm{CaO} \mathrm{C}-\mathrm{TS} 2$ & 27.2 & 29.5 & -3.3 & \\
\hline
\end{tabular}

Table S2. All vibrations of the transition states

\begin{tabular}{llllllll}
\hline \multicolumn{7}{c}{ C-H activation } & \multicolumn{2}{c}{ C-O rebound } \\
\hline & ${ }^{3} \mathrm{O}_{2}-\mathrm{C}-$ & $\mathrm{Al}_{4} \mathrm{CaO}-$ & $\mathrm{Al}_{4} \mathrm{CaO}-$ & $\mathrm{Al}_{4} \mathrm{CaO}_{2}-$ & $\mathrm{Al}_{4}{ }^{2-} \mathrm{O}_{2}-$ & $\mathrm{Al}_{4} \mathrm{CaO}_{2}{ }^{-}$ & $\mathrm{Al}_{4} \mathrm{CaO}-$ \\
& $\mathrm{TS} 1$ & $\mathrm{I}-\mathrm{C}-\mathrm{TS} 1$ & $\mathrm{C}-\mathrm{TS} 1$ & $\mathrm{C}-\mathrm{TS} 1$ & $\mathrm{C}-\mathrm{TS} 1$ & $\mathrm{C}-\mathrm{TS} 2$ & $\mathrm{C}-\mathrm{TS} 2$ \\
\hline Barrier & 111.9 & 71.1 & 67.6 & 52.5 & 42.8 & 32.8 & 27.2 \\
$(\mathrm{kcal} / \mathrm{mol})$ & & & & & & & \\
\hline Frequenci & -1277.6 & -237.4 & -1742.8 & -1293.2 & -950.5 & -460.7 & -252.4 \\
es & 149.0 & 30.2 & 67.1 & 8.6 & 19.5 & 50.4 & 48.6 \\
$\left(\mathrm{~cm}^{-1}\right)$ & 183.4 & 36.7 & 80.5 & 23.1 & 68.3 & 74.0 & 52.5 \\
& 301.4 & 44.4 & 93.7 & 88.4 & 112.3 & 95.6 & 60.8 \\
& 365.0 & 105.3 & 102.5 & 97.8 & 131.9 & 102.6 & 97.1 \\
& 402.7 & 105.8 & 110.3 & 131.6 & 145.5 & 122.4 & 112.2 \\
& 744.7 & 111.4 & 118.9 & 148.1 & 169.8 & 138.6 & 114.1 \\
& 824.7 & 170.5 & 127.2 & 161.8 & 222.9 & 145.5 & 130.3 \\
& 837.2 & 186.3 & 165.7 & 178.7 & 236.9 & 153.2 & 184.0 \\
& 1225.1 & 193.0 & 186.5 & 179.8 & 243.7 & 156.2 & 184.5 \\
& 1418.8 & 194.1 & 197.0 & 218.4 & 256.8 & 177.3 & 224.8 \\
& 1420.9 & 249.9 & 214.5 & 237.1 & 301.2 & 194.1 & 247.9 \\
& 3077.2 & 257.6 & 248.4 & 262.6 & 341.1 & 199.1 & 256.8 \\
& 3234.3 & 259.8 & 271.6 & 275.8 & 397.2 & 206.2 & 290.6 \\
& 3236.5 & 286.2 & 313.1 & 307.4 & 452.5 & 220.1 & 295.7 \\
& & 400.4 & 440.3 & 315.3 & 478.4 & 230.5 & 323.5 \\
& & 403.0 & 458.3 & 383.2 & 518.6 & 377.8 & 387.3 \\
\hline
\end{tabular}




\begin{tabular}{lllllll}
\hline 405.5 & 476.7 & 429.8 & 606.4 & 646.5 & 423.1 \\
800.7 & 567.0 & 463.6 & 658.2 & 661.6 & 505.6 \\
1104.8 & 804.7 & 563.9 & 1042.1 & 667.5 & 711.0 \\
1105.9 & 1188.0 & 615.7 & 1268.8 & 690.2 & 777.2 \\
1424.4 & 1279.8 & 755.3 & 1425.2 & 722.0 & 1403.7 \\
1424.7 & 1420.2 & 1205.5 & 1456.5 & 825.4 & 1412.3 \\
1805.3 & 1423.0 & 1301.5 & 1480.1 & 1032.8 & 3108.6 \\
3030.4 & 3058.2 & 1377.4 & 2881.4 & 1413.5 & 3277.5 \\
3160.5 & 3196.5 & 1486.2 & 2952.6 & 1458.1 & 3294.8 \\
3161.3 & 3201.7 & 1491.8 & 2959.1 & 3079.2 & 3666.9 \\
& & 2865.7 & & 3254.8 & \\
\hline
\end{tabular}

The Z-MATRIX of the optimized structures

1) ${ }^{3} \mathrm{O}_{2}$-C-TS1

$\mathrm{O}$

$\begin{array}{ccccccc}\mathrm{O} & 1 & 1.707397 & & & & \\ \mathrm{C} & 1 & 2.731559 & 2 & 177.517425 & & \\ \mathrm{H} & 3 & 1.084789 & 1 & 99.938671 & 2 & 134.690250 \\ \mathrm{H} & 3 & 1.085074 & 1 & 103.292146 & 2 & 14.365712 \\ \mathrm{H} & 1 & 1.102642 & 2 & 174.050662 & 3 & 6.070229 \\ \mathrm{H} & 3 & 1.084814 & 1 & 100.158335 & 2 & -106.047919\end{array}$

2) $\mathrm{Al}_{4} \mathrm{CaO}-\mathrm{I}-\mathrm{C}-\mathrm{TS} 1$

Al

$\begin{array}{lll}\mathrm{Al} & 1 & 2.686002\end{array}$

$\begin{array}{lllll}\mathrm{Al} & 1 & 2.686625 & 2 & 89.982049\end{array}$

$\begin{array}{lllllll}\mathrm{O} & 2 & 2.153504 & 1 & 52.264681 & 3 & -38.236621\end{array}$

$\begin{array}{lllllll}\mathrm{Al} & 4 & 2.163747 & 2 & 77.135217 & 1 & 127.746670\end{array}$

$\begin{array}{llllrlr}\mathrm{Ca} & 2 & 3.217402 & 1 & 65.956587 & 4 & 101.168435\end{array}$

$\begin{array}{lllllll}\mathrm{C} & 4 & 3.095815 & 2 & 119.649475 & 1 & -117.736872\end{array}$

$\begin{array}{llllrrr}\mathrm{H} & 7 & 1.089395 & 4 & 104.883040 & 2 & 94.054774\end{array}$

$\begin{array}{lllllll}\mathrm{H} & 4 & 1.063590 & 2 & 119.367524 & 1 & -116.624695\end{array}$

$\begin{array}{lllllll}\mathrm{H} & 7 & 1.089386 & 4 & 103.458481 & 2 & -146.005953\end{array}$

$\begin{array}{lllllll}\mathrm{H} & 7 & 1.089478 & 4 & 104.450340 & 2 & -26.270851\end{array}$

3) $\mathrm{Al}_{4} \mathrm{CaO}-\mathrm{C}-\mathrm{TS} 1$

$\mathrm{Al}$

Al $\quad 1 \quad 2.692788$

$\begin{array}{lllll}\mathrm{Al} & 1 & 2.647672 & 2 & 89.812186\end{array}$

$\begin{array}{lllllll}\mathrm{O} & 3 & 1.882529 & 1 & 117.060710 & 2 & 32.607095\end{array}$

$\begin{array}{lllllll}\mathrm{Al} & 4 & 1.885003 & 3 & 96.999831 & 1 & -55.635997\end{array}$

$\begin{array}{lllllll}\mathrm{Ca} & 1 & 3.057936 & 2 & 63.935679 & 5 & -64.427340\end{array}$

$\begin{array}{lllllll}\mathrm{C} & 4 & 3.200053 & 3 & 119.671987 & 1 & -168.587064\end{array}$

$\begin{array}{lllllll}\mathrm{H} & 4 & 1.199999 & 3 & 119.196613 & 1 & -173.087497\end{array}$

$\begin{array}{llllrrr}\mathrm{H} & 7 & 1.085689 & 4 & 107.178160 & 3 & 48.024226\end{array}$

$\begin{array}{lllllll}\mathrm{H} & 7 & 1.086734 & 4 & 100.464994 & 3 & -73.201658\end{array}$

$\begin{array}{lllllll}\mathrm{H} & 7 & 1.086086 & 4 & 98.764961 & 3 & 168.415143\end{array}$

4) $\mathrm{Al}_{4} \mathrm{CaO}_{2}-\mathrm{C}-\mathrm{TS} 1$

$\mathrm{Al}$ 


$\begin{array}{lrrrrrc}\mathrm{O} & 1 & 2.099208 & & & & \\ \mathrm{~A} 1 & 2 & 2.036314 & 1 & 89.032839 & & \\ \mathrm{Al} & 1 & 2.711430 & 2 & 71.337185 & 3 & -107.969073 \\ \mathrm{Al} & 3 & 2.705611 & 2 & 72.851655 & 1 & 108.131236 \\ \mathrm{O} & 2 & 1.504529 & 1 & 106.047126 & 4 & -1.264783 \\ \mathrm{C} & 2 & 2.934265 & 1 & 130.001068 & 4 & 113.714661 \\ \mathrm{H} & 7 & 1.115126 & 2 & 110.482902 & 1 & 44.351239 \\ \mathrm{H} & 7 & 1.108763 & 2 & 109.186313 & 1 & 162.532524 \\ \mathrm{H} & 7 & 1.102639 & 2 & 113.308036 & 1 & -76.956681 \\ \mathrm{H} & 2 & 1.331519 & 1 & 127.511291 & 4 & 113.814439 \\ \mathrm{Ca} & 1 & 3.153572 & 2 & 93.878500 & 6 & 61.658819\end{array}$

5) $\mathrm{Al}_{4}{ }^{2-} \mathrm{O}_{2}-\mathrm{C}-\mathrm{TS} 1$

$\mathrm{Al}$

$\begin{array}{lll}\mathrm{O} & 1 & 2.093375\end{array}$

$\begin{array}{lllll}\mathrm{Al} & 2 & 2.095501 & 1 & 85.166792\end{array}$

$\begin{array}{lllllll}\mathrm{Al} & 1 & 2.720677 & 2 & 71.376398 & 3 & -109.575427\end{array}$

$\begin{array}{lllllll}\mathrm{Al} & 3 & 2.721069 & 2 & 71.349003 & 1 & 109.258903\end{array}$

$\begin{array}{lllllll}\mathrm{O} & 2 & 1.498167 & 1 & 105.289536 & 4 & -4.999020\end{array}$

$\begin{array}{lllllll}\mathrm{C} & 2 & 2.810715 & 1 & 130.313628 & 4 & 115.109226\end{array}$

$\begin{array}{lllllll}\mathrm{H} & 7 & 1.107873 & 2 & 108.436627 & 1 & 41.375571\end{array}$

$\begin{array}{lllllll}\mathrm{H} & 7 & 1.107482 & 2 & 108.564867 & 1 & 159.702756\end{array}$

$\begin{array}{lllllll}\mathrm{H} & 7 & 1.107221 & 2 & 112.308053 & 1 & -79.327877\end{array}$

$\begin{array}{lllllll}\mathrm{H} & 2 & 1.157440 & 1 & 129.271983 & 4 & 114.819313\end{array}$

6) $\mathrm{Al}_{4} \mathrm{CaO}_{2}-\mathrm{C}-\mathrm{TS} 2$

Al

$\begin{array}{llllclc}\mathrm{Al} & 1 & 2.808904 & & & & \\ \mathrm{O} & 2 & 1.767387 & 1 & 37.397001 & & \\ \mathrm{Al} & 1 & 2.853461 & 2 & 89.424745 & 3 & 110.065865 \\ \mathrm{Al} & 4 & 2.783017 & 1 & 90.846265 & 2 & 0.331806 \\ \mathrm{O} & 4 & 2.042965 & 1 & 100.189924 & 2 & -46.631919 \\ \mathrm{C} & 6 & 2.011933 & 4 & 122.793348 & 1 & -39.964416 \\ \mathrm{H} & 7 & 1.081844 & 6 & 98.893712 & 4 & -70.328009 \\ \mathrm{H} & 7 & 1.083066 & 6 & 97.130102 & 4 & 49.669798 \\ \mathrm{H} & 7 & 1.081936 & 6 & 98.816598 & 4 & 169.875304 \\ \mathrm{Ca} & 2 & 3.144349 & 1 & 63.553718 & 3 & -172.678245 \\ \mathrm{H} & 6 & 0.963126 & 4 & 116.220098 & 1 & -161.110057\end{array}$

7) $\mathrm{Al}_{4} \mathrm{CaO}-\mathrm{C}-\mathrm{TS} 2$

Al

$\begin{array}{llllclc}\mathrm{Al} & 1 & 2.719236 & & & & \\ \mathrm{Al} & 2 & 2.734510 & 1 & 88.572281 & & \\ \mathrm{Ca} & 3 & 2.981435 & 2 & 62.707874 & 1 & 68.330610 \\ \mathrm{Al} & 1 & 2.601281 & 2 & 91.436948 & 3 & 0.003307 \\ \mathrm{O} & 3 & 1.975780 & 2 & 46.269052 & 1 & -86.024133 \\ \mathrm{H} & 6 & 0.970794 & 3 & 121.974365 & 2 & 126.579616 \\ \mathrm{C} & 6 & 2.457349 & 3 & 125.401928 & 2 & -135.886034 \\ \mathrm{H} & 8 & 1.078605 & 6 & 88.137649 & 3 & 59.675569 \\ \mathrm{H} & 8 & 1.081152 & 6 & 99.300909 & 3 & -59.276372 \\ \mathrm{H} & 8 & 1.081135 & 6 & 100.514866 & 3 & 178.717000\end{array}$

8) $\mathrm{Al}_{4}{ }^{2-} \mathrm{O}_{2}-\mathrm{C}$

Al

Al

Al

2.718035

$2.736890 \quad 2$

89.866784

$\begin{array}{lllll}2.726746 & 1 & 90.115805 & 2 & -0.249135\end{array}$

$\begin{array}{lllll}2.016813 & 1 & 71.902358 & 2 & -44.914870\end{array}$

$\begin{array}{llllllc}\mathrm{O} & 5 & 1.472482 & 3 & 108.316129 & 1 & -0.081693 \\ \mathrm{C} & 6 & 3.489847 & 5 & 101.519343 & 3 & -138.403526\end{array}$

$\begin{array}{llllllr}\mathrm{C} & 6 & 3.489847 & 5 & 101.519343 & 3 & -138.403526 \\ \mathrm{H} & 7 & 1.093242 & 6 & 108.041460 & 5 & 154.712227\end{array}$

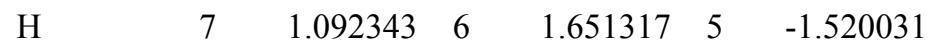




$\begin{array}{llllllr}\mathrm{H} & 7 & 1.093354 & 6 & 109.592517 & 5 & -86.218663 \\ \mathrm{H} & 7 & 1.093404 & 6 & 110.941699 & 5 & 34.727127\end{array}$

9) $\mathrm{Al}_{4} \mathrm{CaO}_{2}-\mathrm{C}$

Al

Al $\quad 1 \quad 2.840456$

$\begin{array}{llll}\mathrm{Al} & 1 & 2.874545 & 2\end{array}$

Al $\quad 3 \quad 2.855238 \quad 1$

$\mathrm{O}-3$

$\mathrm{O} \quad 5$

$\begin{array}{llllr}1.968262 & 1 & 69.083821 & 2 & -39.156812\end{array}$

$\begin{array}{llllr}1.461710 & 3 & 111.015734 & 1 & -0.356955\end{array}$

$\begin{array}{lllllll}\mathrm{C} & 6 & 3.889646 & 5 & 91.360732 & 3 & -140.989656\end{array}$

$\begin{array}{lllllll}\mathrm{H} & 7 & 1.091382 & 6 & 106.908513 & 5 & 149.727010\end{array}$

$\begin{array}{lllllll}\mathrm{H} & 7 & 1.090687 & 6 & 4.946140 & 5 & 25.836492\end{array}$

$\begin{array}{lllllll}\mathrm{H} & 7 & 1.091473 & 6 & 107.330926 & 5 & -93.092870\end{array}$

$\begin{array}{lllllll}\mathrm{H} & 7 & 1.091268 & 6 & 114.488915 & 5 & 28.428400\end{array}$

$\begin{array}{llllrll}\mathrm{Ca} & 4 & 3.235621 & 3 & 63.820406 & 1 & 60.787255\end{array}$

10) $\mathrm{Al}_{4} \mathrm{CaO}-\mathrm{C}$

Al

$\mathrm{Al}$

$\mathrm{Al}$

2.761567

2.700227

$\begin{array}{lllll}1.753013 & 1 & 127.157995 & 2 & 15.529800\end{array}$

$\begin{array}{lllll}1.752803 & 3 & 101.349401 & 1 & -24.951345\end{array}$

$\begin{array}{lllll}3.044540 & 2 & 63.040552 & 5 & -71.085778\end{array}$

$\begin{array}{lllll}3.667467 & 3 & 125.181230 & 1 & 143.655116\end{array}$

$\begin{array}{llllr}1.091339 & 4 & 1.198166 & 3 & 175.006402\end{array}$

$\begin{array}{llllr}1.091767 & 4 & 109.956051 & 3 & 96.212575\end{array}$

$\begin{array}{lllll}1.091823 & 4 & 108.639773 & 3 & -23.245173\end{array}$

$\begin{array}{lllll}1.091758 & 4 & 110.652673 & 3 & -143.061830\end{array}$

11) $\mathrm{Al}_{4} \mathrm{CaO}-\mathrm{I}-\mathrm{C}$

$\mathrm{Al}$

$\mathrm{Al}$

2.670934

$\begin{array}{lll}2.674381 & 2 & 89.994731\end{array}$

$\begin{array}{rrrrr}2.002852 & 1 & 48.237020 & 2 & 26.823534\end{array}$

$\mathrm{O}$

2.008766

$\begin{array}{lll}83.625920 & 1 & -142.565475\end{array}$

$\begin{array}{lllll}3.349626 & 1 & 67.218786 & 2 & -65.169595\end{array}$

$\begin{array}{lllll}4.075112 & 3 & 108.951132 & 1 & 109.862196\end{array}$

$\begin{array}{lllll}1.091558 & 4 & 108.745233 & 3 & -60.539816\end{array}$

$\begin{array}{lllll}1.091064 & 4 & 3.308310 & 3 & 45.076840\end{array}$

$\begin{array}{rrrrr}1.091493 & 4 & 112.851347 & 3 & 60.955168\end{array}$

$\begin{array}{lllll}1.091353 & 4 & 107.144953 & 3 & -178.626859\end{array}$

12) $\mathrm{Al}_{4} \mathrm{CaO}_{2}$-C-int

$\mathrm{Al}$

$\begin{array}{lll}\mathrm{Al} & 1 & 2.956786\end{array}$

$\begin{array}{lll}\mathrm{O} & 2 & 1.912661\end{array}$

Al $\quad 1 \quad 3.325436 \quad 2$

$\mathrm{Al} \quad 4 \quad 3.183709 \quad 1$

$\begin{array}{llll}\mathrm{O} & 5 & 1.747295 & 4\end{array}$

$3.161787 \quad 2$

$\begin{array}{ccccccc}\mathrm{H} & 7 & 1.082226 & 3 & 98.187771 & 2 & -169.155625 \\ \mathrm{H} & 3 & 0.975047 & 2 & 126.915664 & 1 & -155.774589\end{array}$

$\begin{array}{ccccccc}\mathrm{H} & 7 & 1.082226 & 3 & 98.187771 & 2 & -169.155625 \\ \mathrm{H} & 3 & 0.975047 & 2 & 126.915664 & 1 & -155.774589\end{array}$

$\begin{array}{lllllll}\mathrm{H} & 7 & 1.082258 & 3 & 98.415577 & 2 & -48.019505\end{array}$

$\begin{array}{lllllll}\mathrm{H} & 7 & 1.082684 & 3 & 84.869439 & 2 & 71.399121\end{array}$

39.390610

$\begin{array}{lll}92.290619 & 3 & 81.139923\end{array}$

$\begin{array}{lll}87.959761 & 2 & -0.331693\end{array}$

$\begin{array}{llll}24.378536 & 1 & -127.098262\end{array}$

$\begin{array}{lll}125.676589 & 1 & -149.227509\end{array}$

$\begin{array}{ccccccc}\mathrm{H} & 7 & 1.082226 & 3 & 98.187771 & 2 & -169.155625 \\ \mathrm{H} & 3 & 0.975047 & 2 & 126.915664 & 1 & -155.774589\end{array}$

$\begin{array}{lllllll}\mathrm{Ca} & 1 & 3.213730 & 2 & 62.705376 & 3 & 145.845583\end{array}$

13) $\mathrm{Al}_{4} \mathrm{CaO}-\mathrm{C}$-int

$\mathrm{Al}$

$\begin{array}{lll}\mathrm{Al} & 1 & 2.603218\end{array}$

$\begin{array}{lllll}\mathrm{Ca} & 1 & 3.127771 & 2 & 65.551235\end{array}$

$\begin{array}{lllllll}\mathrm{Al} & 1 & 2.687165 & 2 & 91.040200 & 3 & 60.454968\end{array}$ 


$\begin{array}{lcccccc}\mathrm{O} & 4 & 1.921394 & 1 & 110.806906 & 2 & 39.482990 \\ \mathrm{Al} & 5 & 1.923725 & 4 & 90.617320 & 1 & -65.838298 \\ \mathrm{C} & 5 & 3.172589 & 4 & 129.566055 & 1 & 148.602117 \\ \mathrm{H} & 7 & 1.081722 & 5 & 94.315170 & 4 & 78.529561 \\ \mathrm{H} & 7 & 1.081716 & 5 & 93.684794 & 4 & -41.588075 \\ \mathrm{H} & 7 & 1.081813 & 5 & 93.075165 & 4 & -161.423069 \\ \mathrm{H} & 5 & 0.973830 & 4 & 129.565609 & 1 & 148.607883\end{array}$

\section{4) $\mathrm{Al}_{4} \mathrm{CaO}-\mathrm{CH}_{3} \mathrm{OH}$}

Al

$\begin{array}{llllcll}\mathrm{Al} & 1 & 2.847508 & & & & \\ \mathrm{O} & 1 & 2.888513 & 2 & 61.616513 & & \\ \mathrm{H} & 3 & 0.963801 & 1 & 115.337333 & 2 & -106.390930 \\ \mathrm{Al} & 1 & 2.717678 & 2 & 88.342602 & 3 & 124.645526 \\ \mathrm{Al} & 5 & 2.686547 & 1 & 91.453081 & 2 & -0.101662 \\ \mathrm{O} & 6 & 1.754061 & 5 & 40.022577 & 1 & -152.394744 \\ \mathrm{C} & 3 & 1.434920 & 1 & 124.658471 & 2 & 112.164094 \\ \mathrm{H} & 8 & 1.093975 & 3 & 111.303543 & 1 & 81.595242 \\ \mathrm{H} & 8 & 1.093917 & 3 & 111.274486 & 1 & -155.554968 \\ \mathrm{H} & 8 & 1.089538 & 3 & 106.442240 & 1 & -36.993744 \\ \mathrm{Ca} & 1 & 3.075485 & 2 & 62.465276 & 6 & 68.299491\end{array}$

\section{5) $\mathrm{Al}_{4} \mathrm{Ca}-\mathrm{CH}_{3} \mathrm{OH}$}

$\mathrm{Al}$

$\begin{array}{lcccccc}\mathrm{Al} & 1 & 2.608407 & & & & \\ \mathrm{Al} & 2 & 2.659171 & 1 & 92.354290 & & \\ \mathrm{Ca} & 3 & 2.929398 & 2 & 65.124939 & 1 & 66.133210 \\ \mathrm{O} & 3 & 2.104464 & 2 & 111.530099 & 1 & -102.101240 \\ \mathrm{C} & 5 & 1.453106 & 3 & 127.798585 & 2 & -27.563847 \\ \mathrm{H} & 5 & 0.967874 & 3 & 111.867881 & 2 & 116.626478 \\ \mathrm{H} & 6 & 1.090844 & 5 & 110.302195 & 3 & 82.456020 \\ \mathrm{H} & 6 & 1.087730 & 5 & 106.002066 & 3 & -36.223056 \\ \mathrm{H} & 6 & 1.090465 & 5 & 109.471522 & 3 & -155.021998 \\ \mathrm{Al} & 1 & 2.604837 & 2 & 88.743290 & 3 & -2.038478\end{array}$

16) $\mathrm{Al}_{4} \mathrm{CaOH}$

Al

$\begin{array}{llclccc}\mathrm{Al} & 1 & 2.598673 & & & & \\ \mathrm{Ca} & 1 & 3.130200 & 2 & 65.479866 & & \\ \mathrm{Al} & 1 & 2.686642 & 2 & 91.509445 & 3 & 60.216948 \\ \mathrm{O} & 4 & 1.937595 & 1 & 110.893361 & 2 & 39.706416 \\ \mathrm{Al} & 5 & 1.936367 & 4 & 90.201981 & 1 & -64.963430 \\ \mathrm{H} & 5 & 0.963540 & 4 & 125.256679 & 1 & 159.905995\end{array}$

17) $\mathrm{Al}_{4} \mathrm{CaH}_{2} \mathrm{O}$

$\mathrm{Al}$

$\mathrm{Al}$
$\mathrm{Ca}$
$\mathrm{Al}$
$\mathrm{O}$
$\mathrm{Al}$

$\begin{array}{ccccc}2.605349 & & & & \\ 3.020176 & 2 & 69.962062 & & \\ 2.658361 & 2 & 91.915239 & 3 & 58.913961 \\ 2.169335 & 1 & 105.099622 & 2 & 102.914325 \\ 2.605275 & 1 & 89.162883 & 4 & 1.973569 \\ 0.968903 & 4 & 116.278965 & 1 & 18.996631 \\ 0.968874 & 4 & 116.290167 & 1 & -109.733989\end{array}$

18) ${ }^{3}\left(\mathrm{Al}_{4}{ }^{2-} \mathrm{O}_{2}\right)$

$\mathrm{Al}$

$\begin{array}{ll}\mathrm{Al} & \mathrm{Al} \\ \mathrm{Al} & 3 \\ \mathrm{O} & 4 \\ \mathrm{O} & 5\end{array}$

$\begin{array}{ccccc}2.727415 & & & & \\ 3.057872 & 2 & 90.025744 & & \\ 2.727418 & 1 & 89.973965 & 2 & 0.048212 \\ 1.987609 & 3 & 71.920923 & 1 & -36.015027 \\ 1.493347 & 4 & 108.076121 & 3 & 0.030023\end{array}$




$\begin{array}{lllllll}19)^{1}\left(\mathrm{Al}_{4}{ }^{2-} \mathrm{O}_{2}\right) & & & & \\ \mathrm{Al} & & & & & & \\ \mathrm{Al} & 1 & 2.716057 & & & & \\ \mathrm{Al} & 1 & 2.741856 & 2 & 90.694284 & & \\ \mathrm{Al} & 3 & 2.716482 & 1 & 89.199337 & 2 & 1.594518 \\ \mathrm{O} & 4 & 2.017481 & 3 & 47.722529 & 1 & -65.708850 \\ \mathrm{O} & 5 & 1.473762 & 4 & 108.347658 & 3 & 107.410888\end{array}$

20) ${ }^{3}\left(\mathrm{Al}_{4} \mathrm{CaO}_{2}\right)$

$\mathrm{Al}$

$\mathrm{Al}$

2.781259

$\begin{array}{lll}3.310840 \quad 2 & 90.068602\end{array}$

$\begin{array}{lllllll}\mathrm{Al} & 3 & 2.781363 & 1 & 90.045327 & 2 & 0.003808 \\ \mathrm{O} & 4 & 1.971463 & 3 & 70.985617 & 1 & -27.238353\end{array}$

$\begin{array}{lllllll}\mathrm{O} & 5 & 1.495681 & 4 & 109.038888 & 3 & -0.163363\end{array}$

$\begin{array}{lllllll}\mathrm{Ca} & 1 & 3.266516 & 2 & 64.813845 & 5 & 83.162004\end{array}$

21) ${ }^{1}\left(\mathrm{Al}_{4} \mathrm{CaO}_{2}\right)$

$\mathrm{Al}$

Al $\quad 1 \quad 2.863260$

$\mathrm{Al} \quad 1 \quad 2.880866$

$\mathrm{Al} \quad 3-2.848877$

$\mathrm{Ca} \quad 1 \quad 3.234484$

$\mathrm{O} \quad 4 \quad 1.972172 \quad 3$

$\begin{array}{lll}90.149093 \quad 2 & 0.013858\end{array}$

$\begin{array}{llll}2 & 63.741573 & 4 & 60.875979\end{array}$

$\begin{array}{lll}43.761555 & 1 & -58.406340\end{array}$

$\begin{array}{lllllll}\mathrm{O} & 6 & 1.462070 & 4 & 110.962754 & 3 & 113.567919\end{array}$

22) $\mathrm{Al}_{4} \mathrm{CaO}-\mathrm{I}$

$\mathrm{Al}$

Al

2.670507

12.675746

Al $\quad 2 \quad 2.670519$

90.019134

$\begin{array}{lll}90.093948 & 3 & 0.046460\end{array}$

$\begin{array}{lllllll}\mathrm{O} & 3 & 2.001519 & 1 & 48.171792 & 2 & 26.701925\end{array}$

$\begin{array}{lllllll}\mathrm{Ca} & 3 & 3.345962 & 1 & 67.300538 & 2 & -65.358852\end{array}$

23) $\mathrm{Al}_{4} \mathrm{CaO}$

$\mathrm{Al}$

Al

2.758632

$\begin{array}{lll}2.703374 & 2 & 89.555827\end{array}$

$\mathrm{Al}$

O

1.750541

$\begin{array}{lll}126.955404 & 2 & 15.022227\end{array}$

Al

$1.750484 \quad 3$

$\begin{array}{lll}101.892437 & 1 & -24.336725\end{array}$

$\mathrm{Ca}$

$3.043003 \quad 1$

$\begin{array}{lll}63.057821 & 3 & 71.160689\end{array}$ 